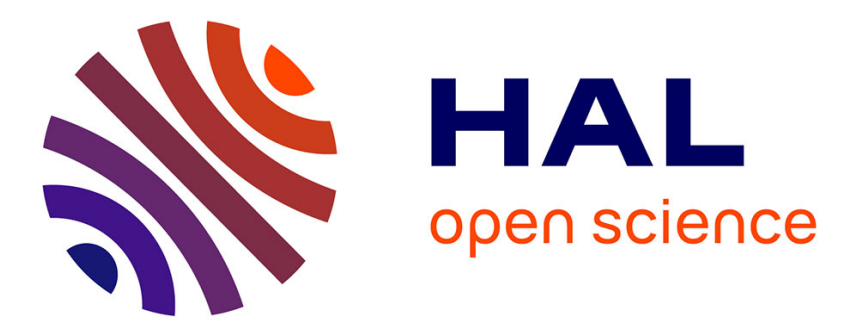

\title{
Cold collisions of SH- with He: Potential energy surface and rate coefficients
}

C T Bop, T Trabelsi, K. Hammami, M Mogren Al Mogren, F. Lique, M. Hochlaf

\section{- To cite this version:}

C T Bop, T Trabelsi, K. Hammami, M Mogren Al Mogren, F. Lique, et al.. Cold collisions of SHwith He: Potential energy surface and rate coefficients. Journal of Chemical Physics, 2017, 147 (12), pp.124301. 10.1063/1.4994970 . hal-03078742

\section{HAL Id: hal-03078742 \\ https://hal.science/hal-03078742}

Submitted on 16 Dec 2020

HAL is a multi-disciplinary open access archive for the deposit and dissemination of scientific research documents, whether they are published or not. The documents may come from teaching and research institutions in France or abroad, or from public or private research centers.
L'archive ouverte pluridisciplinaire HAL, est destinée au dépôt et à la diffusion de documents scientifiques de niveau recherche, publiés ou non, émanant des établissements d'enseignement et de recherche français ou étrangers, des laboratoires publics ou privés. 


\title{
Cold collisions of $\mathrm{SH}^{-}$with He: Potential energy surface and rate coefficients
}

\author{
C. T. Bop, ${ }^{1, a)}$ T. Trabelsi, ${ }^{2}$ K. Hammami, ${ }^{2}$ M. Mogren Al Mogren, ${ }^{3}$ F. Lique,${ }^{4}$ and M. Hochlaf $\left.5, b\right)$ \\ ${ }^{1}$ Laboratory of Atoms Lasers, Department of Physics, Faculty of Sciences and Techniques, University Cheikh \\ Anta Diop of Dakar, Dakar, Senegal \\ ${ }^{2}$ Laboratory of Atomic Molecular Spectroscopy and Applications, Department of Physics, Faculty of Sciences, \\ University Tunis El Manar, Campus Universities, 1060 Tunis, Tunisia \\ ${ }^{3}$ Chemistry Department, Faculty of Science, King Saud University, P.O. Box 2455, Riyadh 11451, \\ Kingdom of Saudi Arabia \\ ${ }^{4}$ LOMC-UMR 6294, CNRS-Université du Havre, 25 Rue Philippe Lebon, BP 1123, \\ 76063 Le Havre Cedex, France \\ ${ }^{5}$ Université Paris-Est, Laboratoire Modélisation et Simulation Multi Echelle, MSME UMR 8208 CNRS, \\ 5 Blvd. Descartes, F-77454 Marne-la-Vallée, France
}

(Received 9 July 2017; accepted 7 September 2017; published online 25 September 2017)

\begin{abstract}
Collisional energy transfer under cold conditions is of great importance from the fundamental and applicative point of view. Here, we investigate low temperature collisions of the $\mathrm{SH}^{-}$anion with He. We have generated a three-dimensional potential energy surface (PES) for the $\mathrm{SH}^{-}\left(\mathrm{X}^{1} \Sigma^{+}\right)-\mathrm{He}\left({ }^{1} \mathrm{~S}\right)$ van der Waals complex. The $a b$ initio multi-dimensional interaction PES was computed using the explicitly correlated coupled cluster approach with simple, double, and perturbative triple excitation in conjunction with the augmented-correlation consistent-polarized valence triple zeta Gaussian basis set. The PES presents two minima located at linear geometries. Then, the PES was averaged over the ground vibrational wave function of the $\mathrm{SH}^{-}$molecule and the resulting two-dimensional PES was incorporated into exact quantum mechanical close coupling calculations to study the collisional excitation of $\mathrm{SH}^{-}$by $\mathrm{He}$. We have computed inelastic cross sections among the 11 first rotational levels of $\mathrm{SH}^{-}$for energies up to $2500 \mathrm{~cm}^{-1}$. (De-)excitation rate coefficients were deduced for temperatures ranging from 1 to $300 \mathrm{~K}$ by thermally averaging the cross sections. We also performed calculations using the new PES for a fixed internuclear $\mathrm{SH}^{-}$distance. Both sets of results were found to be in reasonable agreement despite differences existing at low temperatures confirming that accurate predictions require the consideration of all internal degrees of freedom in the case of molecular hydrides. The rate coefficients presented here may be useful in interpreting future experimental work on the $\mathrm{SH}^{-}$negative ion colliding with $\mathrm{He}$ as those recently done for the $\mathrm{OH}^{-}-\mathrm{He}$ collisional system as well as for possible astrophysical applications in case $\mathrm{SH}^{-}$would be detected in the interstellar medium. Published by AIP Publishing. https://doi.org/10.1063/1.4994970
\end{abstract}

\section{INTRODUCTION}

Inelastic and reactive collisions between ions and neutral species at low temperatures are of fundamental and applicative interests. In the last decades, a huge amount of development, both theoretical and experimental, was achieved in this field since these collisions allow understanding the fundamental processes taking place in chemistry and physics as recently reviewed. ${ }^{1}$

Inelastic collisions under cold conditions have been the object of numerous studies..$^{2-4}$ A particular interest is that molecules can be cooled down through collisions, for example, using experimental techniques such as buffer gas cooling. Such collisions have to be considered using pure quantum approaches since quantum effects are expected to dominate collisional processes at low temperatures.

\footnotetext{
a)Electronic mail: cheikhtidiane.bop@ucad.edu.sn

b)Electronic mail: hochlaf@univ-mlv.fr.
}

Despite recent progress, the case of negative molecular ions colliding with neutrals is still challenging. 5 Whereas, there are no major distinctions between cations and anions colliding with neutrals at a large intermonomer separation, the situation is quite different at short ranges, where the nonreactive scattering of anions strongly depends on the distance and the angle of the approach. ${ }^{5}$ These effects are related to the interaction potentials at the molecular region, where an anionneutral complex may be formed. Moreover, negative ions may exhibit facile autodetachment if their electron affinities either isolated or complexed are not large enough. Autodetachment is in competition with inelastic processes that may occur during these collisions.

In a pioneer work, Wester and co-workers carried out experiments where state-to-state inelastic collisions of hydroxyl anions with helium were studied. The challenging character in these experiments resides on the full quantum state control of the anions, which are hard to manipulate at low energy regimes. For the $\mathrm{OH}^{-}-\mathrm{He}$ benchmark system, they deduced absolute de-excitation rate coefficients. ${ }^{6}$ The 
assignments of the observed features were supported by quantum scattering theory calculations. However, the theoretical findings did not match perfectly with the experimental ones showing that more theoretical and experimental studies need to be performed to have a better insight in these processes. Indeed, the corrigendum of Ref. 6 shows that the computed data are smaller than the measured ones.

Cold inelastic collisions are also widely present in astrophysical media such as in the interstellar medium (ISM), where they contribute to the excitation of the interstellar species. Several studies were performed in order to help astronomers in the accurate determination of the molecular content of the ISM. ${ }^{7}$

Most of the detected species are neutrals or positively charged ions. Only few negative ions were recently identified. They correspond to negative carbon chains such as $\mathrm{C}_{2 n+1} \mathrm{~N}^{-}$ $(n=0-2)^{8-10}$ and $\mathrm{C}_{2 n+2} \mathrm{H}^{-}(n=1-3) .{ }^{1-13}$ They were observed toward the carbon rich star IRC +10216 , the Low-Mass Protostar IRAS $04368+2557$ in L1527, and the cold dark TMC-1 cloud. The reasons for such a very low number of anions present in the ISM were attributed to their favorable autodetachment via non-adiabatic effects and/or after reactive collisions with surrounding atoms, molecules, and substrates hence forming neutral species. ${ }^{14}$

Here, we are interested in the cold collision implying the $\mathrm{SH}^{-}$anion and the He atom. The $\mathrm{SH}^{-}$anion has a large positive electron affinity $\left(\sim 2.31 \mathrm{eV}^{15}\right)$. It is thus a stable species in the gas phase. Interaction between $\mathrm{He}$ and $\mathrm{SH}^{-}$can also be a good candidate for future scattering experiments such as those performed recently on the $\mathrm{OH}^{-}-\mathrm{He}$ system. ${ }^{6}$ In addition, its neutral and cation associated species, $\mathrm{SH}$ and $\mathrm{SH}^{+}$, were recently detected in space. ${ }^{16,17} \mathrm{SH}^{-}$can then be a constituent of the ISM and joins the list of S-bearing diatomic molecules that were already detected: $\mathrm{CS},{ }^{18} \mathrm{SO},{ }^{19} \mathrm{NS},{ }^{20} \mathrm{SiS},{ }^{21}$ and $\mathrm{SO}^{+} .{ }^{22}$ In the case of the detection of $\mathrm{SH}^{-}$, collisional rate coefficients for the $\mathrm{SH}^{-}-\mathrm{He}$ system will be of primary interest for the analysis of the interstellar spectra.

In this paper, we first mapped the $\mathrm{SH}^{-}-\mathrm{He}$ interaction potential in the Jacobi coordinates (see Fig. 1). The electronic structure computations were performed using explicitly correlated coupled cluster approach. A full three dimensional

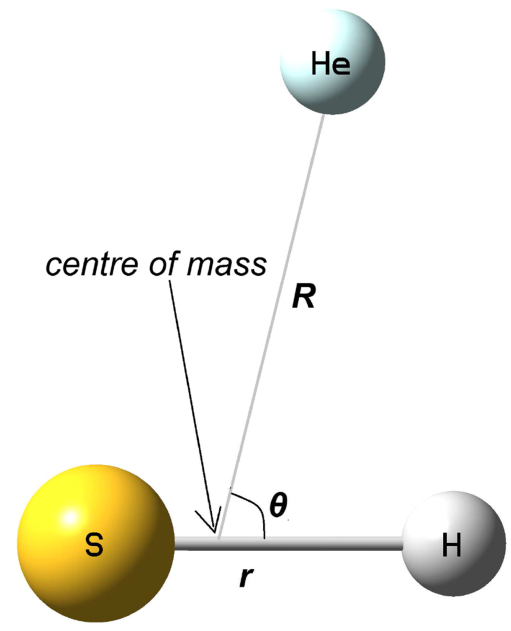

FIG. 1. Body-fixed Jacobi coordinates of the $\mathrm{SH}^{-}-\mathrm{He}$ collisional complex. potential energy surface (3D-PES) was generated. Afterwards, the 3D-PES averaged over the ground vibrational wave function of $\mathrm{SH}^{-}$was incorporated into quantum dynamical calculations to deduce the rotational (de-)excitation cross sections of $\mathrm{SH}^{-}$colliding with $\mathrm{He}$ and the corresponding rate coefficients. The results were compared with those obtained from the same PES but for a fixed internuclear $\mathrm{SH}^{-}$distance. Hence, we will check on the accuracy of the use of interaction potentials calculated within the rigid rotor (RR) approximation for solely the experimental equilibrium distance of the molecule (i.e., the accuracy of pure 2D-PESs).

The present data should help in interpreting corresponding experimental data whenever measured. As pointed out above, collisional data were rarely measured for negative ions and theoretical computations such as those performed here represent precious help with respect to this objective. Finally, we will carry out a comparison with the $\mathrm{OH}^{-}-\mathrm{He}$ isovalent system for which both theoretical and experimental data are available. ${ }^{6,23}$

This paper is organized as follows: Sec. II presents the potential energy surfaces along with the computational details. Section III presents our dynamical computations data and the comparison to the $\mathrm{OH}^{-}-\mathrm{He}$ system. In Sec. IV, some concluding remarks are drawn.

\section{POTENTIAL ENERGY SURFACE}

To compute the $\mathrm{SH}^{-}-\mathrm{He}$ interaction potential, preliminary computations were first performed using the complete active space self-consistent field method (CASSCF) ${ }^{24,25}$ to check on the monoconfigurational character of the $\mathrm{SH}^{-}-\mathrm{He}$ van der Waals system over a large range of internuclear configurations. These computations showed that the dominant electronic configuration of the ground electronic state of the $\mathrm{SH}^{-}-\mathrm{He}$ system has a weight greater than $99.50 \%$. Hence, we followed the methodology that we established for the generation of potentials using explicitly correlated calculations. Briefly, the calculations were carried out using the explicitly correlated coupled cluster approach with single, double and perturbative triple excitation $\operatorname{CCSD}(\mathrm{T})-\mathrm{F} 12^{26}$ as implemented in the Molpro suite (version 2012). ${ }^{27}$ The atoms were described with the augmented-correlation consistent-polarized valence triple zeta (aug-cc-pVTZ) basis set. ${ }^{28,29}$ The MOLPRO default choices for the density fitting and resolution of identity basis sets have been applied. ${ }^{30}$ As we established, ${ }^{31-34}$ this level of theory ensures taking into account large part of electronic correlation similar to that reached by using the standard coupled cluster technique extrapolated to the complete basis set (CBS) limit.

We computed a potential energy surface modeling the interactions between a diatomic anion and a structureless neutral atom. For related studies, the internuclear distance of the diatom is usually kept frozen at its experimental value. Nevertheless, a 3D-PES is more suited if molecules are prepared in a vibrationally excited state, which is of interest to interpret future possible experiments. In addition, it was recently established that an accurate description of the target vibrational motion in the van der Waals complex is needed for low energy scattering. ${ }^{35}$ Indeed, there is a need to accurately take into account the vibrational motion of diatomic 
molecules in the construction of potential energy surfaces, especially those implying molecular hydrides where a significant anisotropy with respect to the internuclear distance $r$ is expected.

Therefore, we generated a 3D-PES of $\mathrm{SH}^{-}$interacting with $\mathrm{He}$. In the calculations, the $\mathrm{SH}^{-}-\mathrm{He}$ van der Waals system was described with the Jacobi coordinates $r, R$, and $\theta: r$ represents the internuclear distance of $\mathrm{SH}^{-}, R$ is the scattering distance (i.e., the distance linking the centre of mass of $\mathrm{SH}^{-}$ and $\mathrm{He}$ ), and $\theta$ stands for the angle that form the two distance vectors (see Fig. 1).

The 3D-PES, V(r, R, $\theta)$, was calculated by setting the $r$ distance to 5 values $\left(2.13,2.33,2.53,2.72\right.$, and $\left.2.92 \mathrm{a}_{0}\right)$ leading to consider the vibrational states of the $\mathrm{SH}^{-}$negative ion up to the vibrational level $v=2$. The scattering $R$-distance was varied from 3.20 to $100 \mathrm{a}_{0}$ with an irregular step. Typically, we considered 37 R-values $(3.2,3.4,3.6,3.84,4.2,4.4,4.6,4.8$, 5.0, 5.2, 5.4, 5.6, 5.8, 6.0, 6.2, 6.4, 6.6, 6.8, 7.0, 7.2, 7.4, 7.6, $7.8,8.0,8.4,8.8,9.0,9.5,10,14,18,20,25,30,40,50$, and $\left.100 \mathrm{a}_{0}\right)$. The angular Jacobi coordinate $\theta$ was spanned with an uniform step size of $10^{\circ}$ from $0^{\circ}$ to $180^{\circ}$. The $3 \mathrm{D}$-PES was constructed in the $C_{s}$ symmetry group with 3515 ab initio energies for non-redundant nuclear configurations. Note that we corrected for the non-size consistency error of the CCSD(T)-F12 approach by subtracting the value of the potential $\sim 1.14 \mathrm{~cm}^{-1}$ obtained at $100 \mathrm{a}_{0}$. This ensures a vanishing potential at large intermonomer separations and hence to a correct asymptotic behavior of the interaction potential. In addition, we have corrected the basis set superposition error (BSSE) with the counterpoise procedure of Boys and Bernardi, ${ }^{36}$

$$
V(r, R, \theta)=E_{S H^{-}-H e}(r, R, \theta)-E_{S H^{-}}(r, R, \theta)-E_{H e}(r, R, \theta),
$$

where $E_{S H^{-}-H e}(r, R, \theta), E_{S H^{-}}(r, R, \theta)$, and $E_{H e}(r, R, \theta)$ are the total electronic energies of the $\mathrm{SH}^{-}-\mathrm{He}$ cluster, the $\mathrm{SH}^{-}$subsystem, and the He subsystem, respectively. These energies are evaluated in the full basis set of the complex.

For dynamical calculations, an analytical expansion of the potentials is required. We have adopted the fitting procedure described by Werner et al. $^{37}$ for the $\mathrm{CN}-\mathrm{He}$ complex. Such a procedure leads us to generate the $V(r, R, \theta)$ numerical expansion routine that is implemented later in the dynamical computations code,

$$
V(r, R, \theta)=\sum_{n=1}^{N} \sum_{l=1}^{L} A_{l n}(R) d_{0,0}^{l-1}(\cos \theta)\left(r-r_{e}\right)^{n-1},
$$

where $d_{0,0}^{l-1}$ are the reduced rotation matrix elements of Wigner, and $\mathrm{L}$ and $\mathrm{N}$ represent, respectively, the numbers of the scattering angles and the $\mathrm{SH}^{-}$internuclear distances. The analytical potential is found to reproduce the calculated surface quite well. The deviations between the fitted potential values and the $a b$ initio points are concentrated in the repulsive part of the PES, and the mean difference between the analytical fit and the $a b$ initio calculations is $\sim 2 \%-3 \%$.

The calculated $a b$ initio 3D-PES was averaged over the ground vibrational wave function $(v=0)$ of the $\mathrm{SH}^{-}$molecule. Thus, we have determined the averaged 3D-PES with the following algorithm:

$$
\begin{gathered}
V(R, \theta)=\left\langle\varphi_{v=0}(r)|V(r, R, \theta)| \varphi_{v^{\prime}=0}(r)\right\rangle, \\
V(R, \theta)=\sum_{n=1}^{N} \sum_{l=1}^{L} A_{l n}(R) d_{0,0}^{l-1}(\cos \theta) \times M_{0,0}(n), \\
M_{v, v^{\prime}}(n)=\left\langle\varphi_{v}(r)\left|\left(r-r_{e}\right)^{n-1}\right| \varphi_{v^{\prime}}(r)\right\rangle .
\end{gathered}
$$

The $\varphi_{v}$ wave functions were computed using the Fourier Grid Hamiltonian (FGH) program ${ }^{38,39}$ from the potential of $\mathrm{SH}^{-}\left(\mathrm{X}^{1} \Sigma^{+}\right)$which was calculated with a RKR program ${ }^{40}$ using the $\mathrm{SH}^{-}$experimental constant of Ref. 41. The vibrational wave
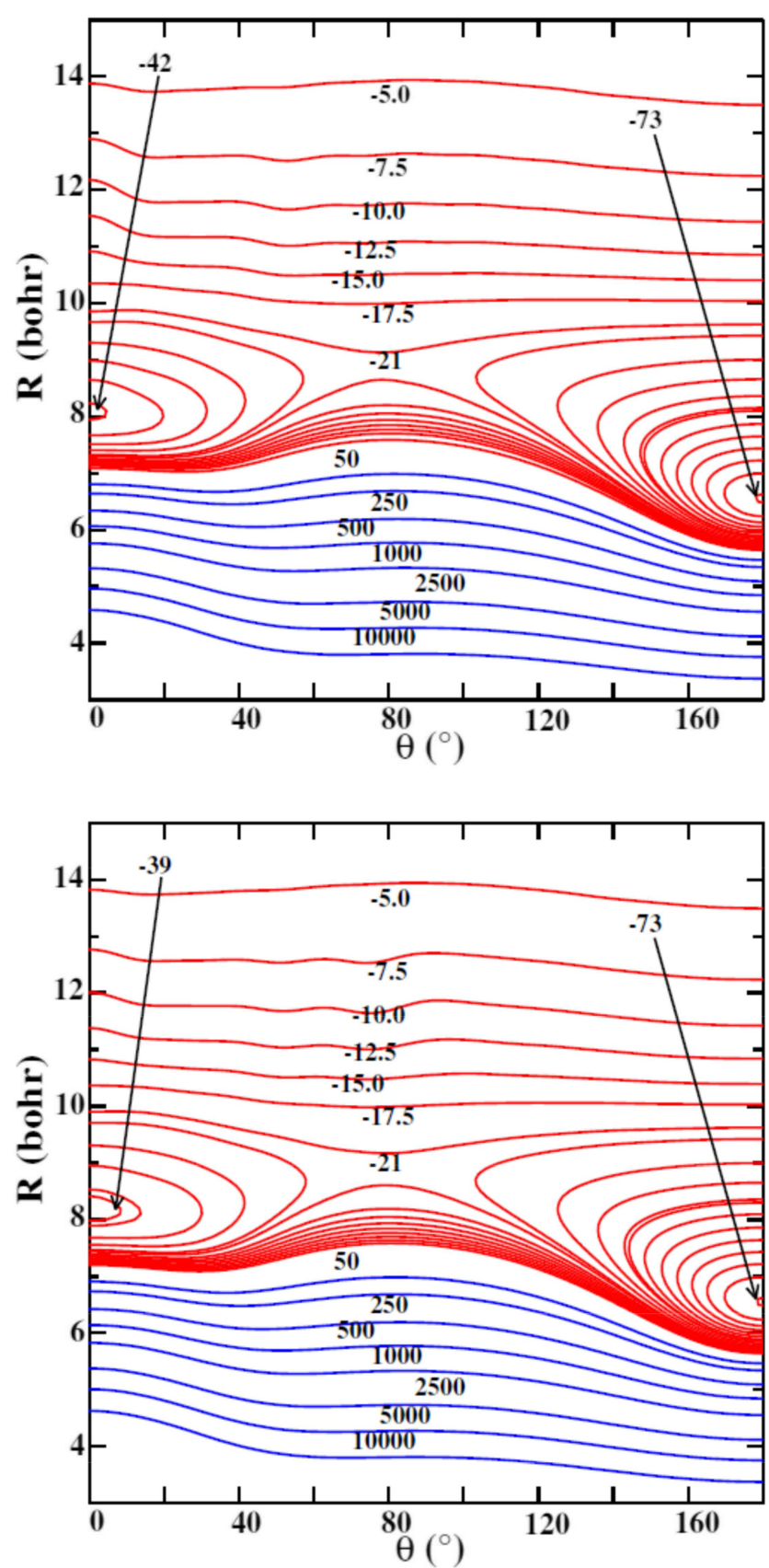

FIG. 2. Contour plots of the $\mathrm{SH}^{-}-\mathrm{He}$ interaction potentials as a function of the $\mathrm{R}$ and $\theta$ Jacobi coordinates: the 3D-PES for a fixed $\mathrm{r}$ distance of 2.53 bohrs (upper panel) and vibrationally averaged 3D-PES (i.e., av-2D-PES) (lower panel). The blue (red) curves stand for positive (negative) energies (in unit of $\mathrm{cm}^{-1}$ ). The zero energy is obtained when $\mathrm{SH}^{-}$and $\mathrm{He}$ are separated. 
function taken for the rotational state $J=0$. The resulting PES is denoted hereafter as av-2D-PES.

Figure 2 displays the 3D-PES (upper panel) at a fixed $\mathrm{SH}^{-}$internuclear distance of $r=2.53$ bohrs (corresponding to the equilibrium distance of the $\mathrm{SH}^{-}$molecule) and the av2D-PES (lower panel). These contour plots of the $\mathrm{SH}^{-}-\mathrm{He}$ van der Waals system are given as a function of the $R$ and $\theta$ Jacobi coordinates. Both interaction potentials are anisotropic and present two minima located at linear configurations (i.e., $\theta=0^{\circ}$ and $\theta=180^{\circ}$ ). These two minima are separated by a transition state for $\theta$ close to $80^{\circ}$. For av-2D-PES, the deepest potential well occurs at $73.11 \mathrm{~cm}^{-1}$ for He approaching toward S (i.e., $\theta=180^{\circ}$ ) and $R=6.6$ bohrs. The depth of the second minimum is $39.86 \mathrm{~cm}^{-1}$ and it is located at $\mathrm{R}=8.2$ bohrs. The well depths of the 2D-PES at a fixed $\mathrm{SH}^{-}$internuclear distance are located at almost the same positions. They are however slightly deeper $\left(73.18 \mathrm{~cm}^{-1}\right.$ for $\theta=180^{\circ}$ and $42.42 \mathrm{~cm}^{-1}$ for $\theta=0^{\circ}$ ). One can conclude from this comparison that both potentials present close shapes in the intermediate region of the scattering distance. However, some deviations are observed in the short range part of the PESs, particularly for $\theta$ $=0^{\circ}-10^{\circ}$.

With respect to the $\mathrm{OH}^{-}-\mathrm{He}$ system, both potentials present two minima in the linear geometries and the deeper one occurs for the He approach toward the heavier atom of the anion (i.e., $\mathrm{S}$ for $\mathrm{SH}^{-}$and $\mathrm{O}$ for $\mathrm{OH}^{-}$, see Table I). However, the anisotropies of the two interaction potentials are different since the minimum observed for $\mathrm{He}$ approaching toward $\mathrm{H}$ (the lighter atom) is deeper for $\mathrm{SH}^{-}$than that for $\mathrm{OH}^{-}$(while the other minimum is deeper for $\mathrm{OH}^{-}$).

In 2D and av-2D PESs, the saddle point for the T-shape geometry is $-21 \mathrm{~cm}^{-1}$ below the $\mathrm{He}-\mathrm{SH}^{-}$PES asymptote. Therefore, large amplitude motions or quantum vibrational localizations for the rovibrational states located above this barrier are expected. Recently, such effects were identified for $\mathrm{SH}^{-}-\mathrm{N}, \mathrm{SN}^{-}-\mathrm{H},{ }^{42}$ and $\mathrm{NO}^{+}-\mathrm{Ar}^{43}$ weakly bound complexes. These effects are worth probing by means of experimental setups.

The influence that the difference mentioned above may carry on dynamical calculations will be presented and discussed in Sec. III. To this end, we have implemented the potentials in the MOLSCAT code. ${ }^{44}$ Both interaction potentials have been expanded over the Legendre polynomial functions by including terms up to $\lambda_{\max }=18$ such as

$$
V(R, \theta)=\sum_{\lambda} V_{\lambda}(R) P_{\lambda}(\cos \theta) .
$$

The $\mathrm{SH}^{-}-\mathrm{He} \mathrm{PES}$ and the $\mathrm{SH}^{-}$potential energy curves can be found in the supplementary material.

TABLE I. Potential wells (in units of $\mathrm{cm}^{-1}$ ) of the $\mathrm{SH}^{-}-\mathrm{He}$ and $\mathrm{OH}^{-}-\mathrm{He}$ systems. For $\mathrm{OH}^{-}-\mathrm{He}$, these values were estimated from Ref. 23.

\begin{tabular}{lccc}
\hline \hline Geometry & \multicolumn{2}{c}{ This work } & Reference 23 \\
\hline $\boldsymbol{\theta}$ (deg) & 2D-PES & av-2D-PES & $\mathrm{OH}^{-}-\mathrm{He}$ \\
0 & 42.42 & 39.86 & $\geq 25$ \\
180 & 73.18 & 73.11 & $\geq 100$ \\
\hline \hline
\end{tabular}

\section{COLLISIONAL DYNAMICS}

One of the objectives of the present work is the determination of (de-)excitation cross sections and rate coefficients for the negative ion $\mathrm{SH}^{-}$in collision with He. All dynamical calculations were carried out with the 3D-PES at a fixed internuclear distance and with the av-2D-PES using the same parameters to probe the impact of the vibration in the magnitude of the collisional data.

\section{A. Rotational cross sections}

In this paper, we only consider the pure rotational excitation of $\mathrm{SH}^{-}$induced by collision with $\mathrm{He}$. The collisional energy range considered in this paper (up to $2500 \mathrm{~cm}^{-1}$ ) is below the first excited vibrational level of $\mathrm{SH}^{-}\left(w_{e}\right.$ $\left.=2647.07 \mathrm{~cm}^{-1}\right)^{45}$ so that vibrational excitation is not likely to occur and can be omitted. Note that the 3D-PES we generated can be used for those purposes if needed.

The excitation cross sections calculations were performed using the quantum mechanical close-coupling (CC) approach of Arthurs and Dalgarno ${ }^{46}$ as implemented in the MOLSCAT program. In the following, $J$ denotes the rotational state of the $\mathrm{SH}^{-}$anion. The total energy range was spanned as follows: for $\mathrm{E} \leq 100 \mathrm{~cm}^{-1}$, the step was set to $0.1 \mathrm{~cm}^{-1}$; for $100 \leq \mathrm{E}$ $\leq 200 \mathrm{~cm}^{-1}$, the step was set to $0.5 \mathrm{~cm}^{-1}$; for $200 \leq \mathrm{E} \leq 500$ $\mathrm{cm}^{-1}$, the step was set to $1 \mathrm{~cm}^{-1}$; for $500 \leq \mathrm{E} \leq 1000 \mathrm{~cm}^{-1}$, the step was set to $5 \mathrm{~cm}^{-1}$; for $1000 \leq \mathrm{E} \leq 1500 \mathrm{~cm}^{-1}$, the step was set to $10 \mathrm{~cm}^{-1}$; and for $1500 \leq \mathrm{E} \leq 2500 \mathrm{~cm}^{-1}$, the step was set to $20 \mathrm{~cm}^{-1}$. The rotational levels of $\mathrm{SH}^{-}$are obtained from the spectroscopic constants reported in Table II. We have performed some convergence tests to set up the MOLSCAT parameters. The selected parameters are given in Table II. The STEPS integration parameter which is inversely proportional to the integration step was set to 20 for $\mathrm{E} \leq 100 \mathrm{~cm}^{-1}$ and 10 for the other energy values. The integration limits are set to $R_{\min }$ $=2$ bohrs and $R_{\max }=90$ bohrs. The size of the rotational basis defined by $J_{\max }$ was set to 7 for total energy $E \leq 100 \mathrm{~cm}^{-1}$ and progressively increased to $J_{\max }=17$ for energy up to $2500 \mathrm{~cm}^{-1}$. In addition, for elastic (inelastic) transitions, the diagonal (off-diagonal) tolerance was set to DTOL $=0.01 \AA^{2}$ (OTOL $=0.001 \AA^{2}$ ). This allows setting the limit value of the total angular momentum $\left(J_{t o t}\right)$ large enough for a best convergence of cross sections. For example, we have obtained $J_{t o t}$ $=157$ at $100 \mathrm{~cm}^{-1}, J_{\text {tot }}=236$ at $1000 \mathrm{~cm}^{-1}$, and $J_{\text {tot }}=280$ at $2500 \mathrm{~cm}^{-1}$. Such a high number was needed for convergence of the elastic cross sections. The log derivative method was used to solve the coupled equations. ${ }^{47}$

Figure 3 displays the rotational de-excitation cross sections of $\mathrm{SH}^{-}$induced by collision with $\mathrm{He}$ as a function of

TABLE II. MOLSCAT parameters used in this work.

\begin{tabular}{lc}
\hline \hline STEPS $=20,10$ & $J_{\max }=7,8,10,13,15,16,17$ \\
\hline$D_{e}=4.984 \times 10^{-4} \mathrm{~cm}^{-1}, 45$ & $R_{\min }=2 a_{0}$ \\
$B_{e}=9.56253 \mathrm{~cm}^{-1}, 45$ & $R_{\max }=90 a_{0}$ \\
DTOL $=0.01 \AA^{2}$ & OTOL $=0.001 \AA^{2}$ \\
$\mu=3.57 \mathrm{amu}$ & \\
\hline
\end{tabular}



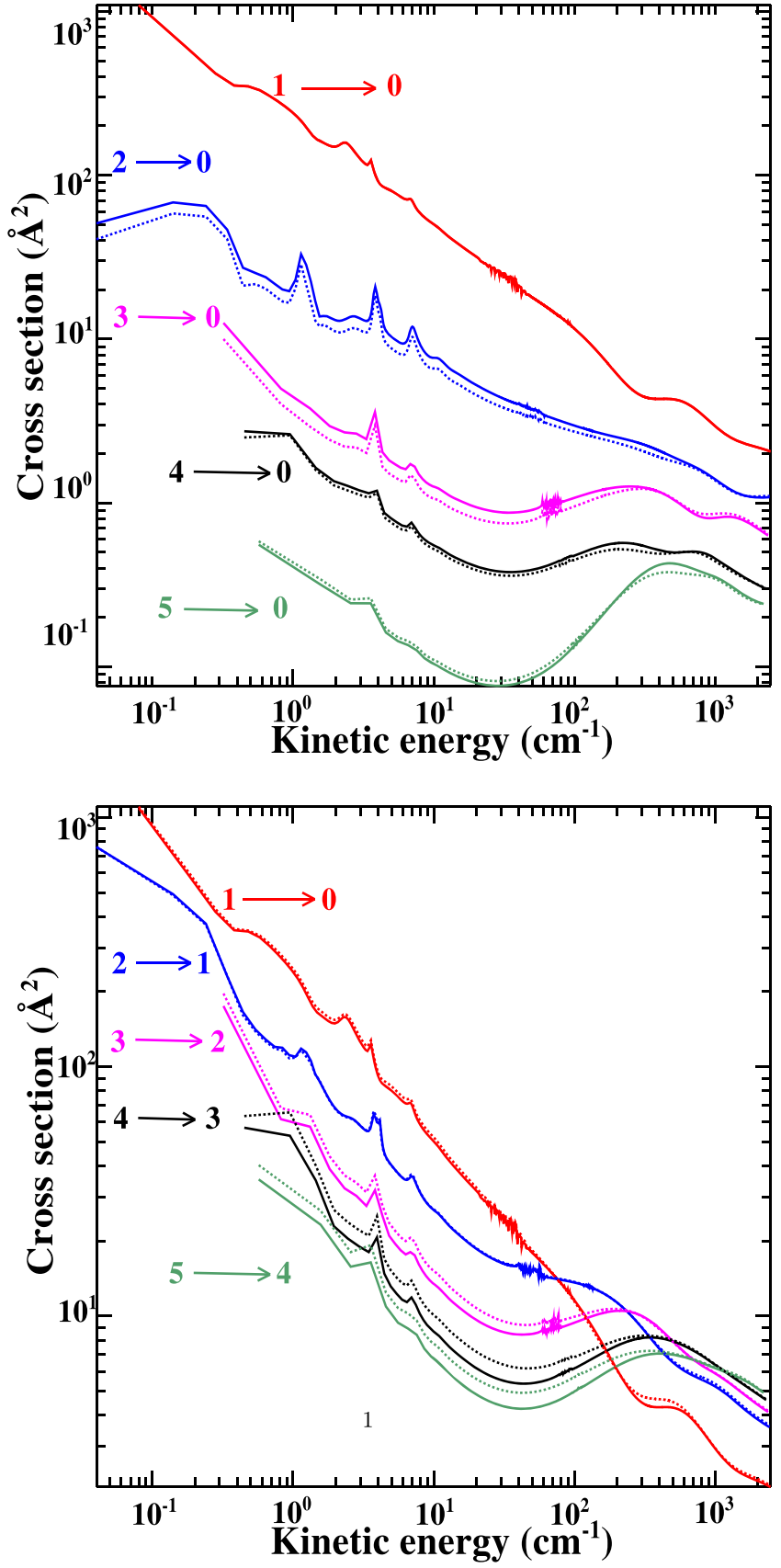

FIG. 3. Rotational de-excitation cross sections of $\mathrm{SH}^{-}$induced by collision with $\mathrm{He}$ as a function of the kinetic energy for $J \rightarrow 0$ (upper panel) and $\Delta J=1$ (lower panel) transitions. The dotted lines (solid lines) correspond to cross sections obtained from the vibrationally averaged av-2D (pure two dimensional) PES.

the kinetic energy for $J \rightarrow 0$ (upper panel) and $\Delta J=1$ (lower panel) transitions. The dotted lines correspond to data obtained from the av-2D-PES and the solid lines correspond to data obtained from the pure 2D-PES. For the $1 \rightarrow 0$ and $2 \rightarrow 1$ transitions, both potentials lead to very close cross section values while deviations are observed in the $2-5 \rightarrow 0,3 \rightarrow 2,4 \rightarrow 3$, and $5 \rightarrow 4$ transitions. When the collision energy increases, the difference between the two sets of results decreases. These differences reflect a slightly different anisotropy of the two PESs. Such conclusion was already addressed for the $\mathrm{OH}-\mathrm{He}$ neutral system by Kalugina et al. ${ }^{35}$ who found that the use of a vibrationally averaged 3D-PES is desired to investigate the excitation of diatomic hydrides at very low energies. In detail, the differences remain however small, and the pure rotational excitation of $\mathrm{SH}^{-}$induced by collision with $\mathrm{He}$ can be reasonably well described by the use of a pure 2D PES. Recently, Faure et $a l .{ }^{48}$ investigated in detail the impact of the vibration of the target on the computation of pure rotational excitation for the $\mathrm{CO}-\mathrm{H}_{2}$ system. These authors found that the use of a rigid rotor PES is reliable when the equilibrium distance is corresponding to the vibrationally averaged distance. For $\mathrm{SH}^{-}$, this would correspond to an internuclear distance of 2.57 $a_{0}$. Hence, we may expect a better agreement between results obtained from pure 2D and av-2D PESs if the equilibrium distance is $2.57 a_{0}$.

Some resonances can be observed in these cross sections at low energies. This behavior was noted in early studies as well as in the recent ones., ${ }^{2,49,50}$ They are of either shape or Feshbach nature. The Feshbach resonances were interpreted as a temporary trapping of helium in the potential well, where the complex $\left(\mathrm{SH}^{-}-\mathrm{He}\right)$ forms quasi-bound states and shape resonances correspond to quasi-bound states occurring from tunneling via the centrifugal energy barrier. The fine step size used at energies below $100 \mathrm{~cm}^{-1}$ was crucial for a correct description of these resonances.

Concerning propensity rules, the $1 \rightarrow 0$ transition predominates in the two panels at low collision energies. At higher collision energies, transitions implying highest rotational states start to dominate. From the analysis of these curves, one can conclude that transitions involving $\Delta J=1$ values outweigh the $\Delta J=2$ ones. We anticipate that this propensity rule persists in the collisional rates.

\section{B. Rate coefficients}

The rate coefficients were computed for temperatures ranging from 1 to $300 \mathrm{~K}$ for all energy levels with $J \leq 10$, by thermally averaging the cross sections over a MaxwellBoltzmann velocity distribution,

$k_{J \rightarrow J^{\prime}}(T)=\left(\frac{8}{\pi \mu k_{B}^{3} T^{3}}\right)^{\frac{1}{2}} \int_{0}^{\infty} \sigma_{J \rightarrow J^{\prime}} E_{c} \exp \left(-\frac{E_{c}}{k_{B} T}\right) d E_{c}$,

where $\sigma_{J \rightarrow J^{\prime}}$ is the cross section from initial state $J$ to final state $J^{\prime}, \mu$ is the $\mathrm{SH}^{-}-\mathrm{He}$ reduced mass, and $k_{B}$ is Boltzmann's constant.

We present in Fig. 4 the $\mathrm{SH}^{-}$de-excitation rate coefficients induced by collision with $\mathrm{He}$ as a function of the kinetic temperature for $J \rightarrow 0$ (upper panel) and $\Delta J=1$ (lower panel) transitions. The curves obtained using the av-2D-PES are plotted with dotted lines, whereas solid lines represent those computed using the pure 2D-PES for a unique $\mathrm{SH}^{-}$internuclear distance (of 2.53 bohrs). As anticipated, small deviations occur between the two sets of data. At high temperatures, the difference between both PESs is small enough to validate the use of a pure 2D-PES in scattering calculations. The upper panel confirms that the propensity rules are in favor of $\Delta J=1$ transitions.

Finally, Table III gives the rate coefficients of both isovalent negative ions $\left(\mathrm{SH}^{-}\right.$and $\left.\mathrm{OH}^{-}\right)$induced by collision with He at low temperatures for comparison. The data obtained 

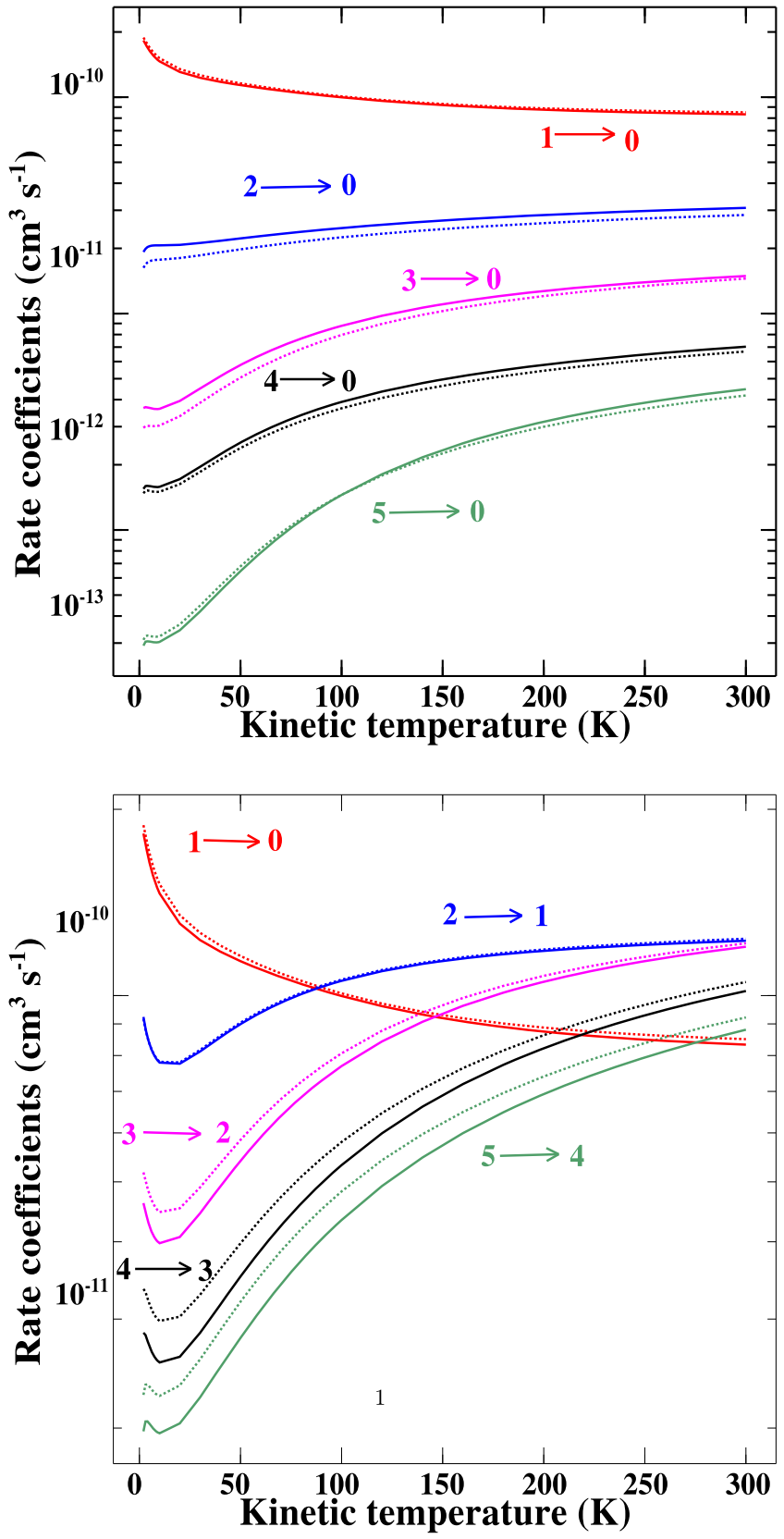

FIG. 4. Rate coefficients of $\mathrm{SH}^{-}$induced by collision with $\mathrm{He}$ as a function of the kinetic temperature for $J \rightarrow 0$ (upper panel) and $\Delta J=1$ (lower panel) transitions. The dotted lines (solid lines) are obtained using av-2D-PES (the 3D-PES for a fixed $r$ distance of 2.53 bohrs).

in this work for $\mathrm{SH}^{-}-\mathrm{He}$ system are $\sim 7.4$ times greater than those measured for $\mathrm{OH}^{-}-\mathrm{He} .^{6}$ Two main reasons can explain this difference: (i) the smaller energy spacing between $\mathrm{SH}^{-}$ rotational states than that between $\mathrm{OH}^{-}$rotational states and

TABLE III. Comparison of rotational de-excitation rate coefficients $\left(\times 10^{-10}\right.$ $\mathrm{cm}^{3} \mathrm{~s}^{-1}$ ) of the $\mathrm{SH}^{-}$and $\mathrm{OH}^{-}$isovalent negative ions induced by collision with $\mathrm{He}$ at low temperatures. All data correspond to the $1 \rightarrow 0$ transition. The $\mathrm{SH}^{-}$data are those obtained from the av-2D PES.

\begin{tabular}{lccc}
\hline \hline$T(\mathrm{~K})$ & $k_{\mathrm{SH}^{-}}$ & $k_{\mathrm{OH}^{-}}$ & $k_{\mathrm{SH}^{-}} / k_{\mathrm{OH}^{-}}$ \\
\hline 22 & 1.328 & $0.180^{\mathrm{a}}$ & 7.378 \\
\hline \hline
\end{tabular}

${ }^{a}$ Refer to data of Hauser et al. ${ }^{6}$ (ii) the different anisotropy of the PESs of the two systems. Hence, we expect that experiments on the $\mathrm{SH}^{-}-\mathrm{He}$ system could also lead to the accurate measurement of the rotational rate coefficients at low temperatures because of a possible strong signal.

\section{CONCLUSION}

We have computed a multi-dimensional PES for the $\mathrm{SH}^{-}-\mathrm{He}$ van der Waals system at the $\operatorname{CCSD}(\mathrm{T})-\mathrm{F} 12 / \mathrm{aug}-$ cc-pVTZ level of theory. The PESs were incorporated into quantum mechanical computations to deduce the state-tostate rotational (de-)excitation cross sections of $\mathrm{SH}^{-}$by He. These cross sections are obtained for total energies up $2500 \mathrm{~cm}^{-1}$. Using a Maxwell-Boltzmann velocity distribution, we have thermally averaged these cross sections and obtained rate coefficients for temperatures ranging from 1 to $300 \mathrm{~K}$.

In this work, we note that the pure two-dimensional potential is well suited to deduce the rotational cross sections and rate coefficients. Indeed, our results show that the $\mathrm{SH}^{-}$vibrational motion does have not a major effect on the scattering process. However, slight deviations occur at low temperatures between the two sets of results obtained with pure two-dimensional and av-2D PESs. This difference may be seen in future highly accurate experimental studies.

Concerning propensity rules, the $\Delta J=1$ transitions are favored compared to the $\Delta J=2$ ones. It is expected that the data computed in this paper may be of great interest for future experimental studies as well as the interstellar detection of the $\mathrm{SH}^{-}$negative ion.

\section{SUPPLEMENTARY MATERIAL}

See supplementary material for the analytical forms of the $\mathrm{SH}^{-}-\mathrm{He}$ potential energy surface and the $\mathrm{SH}^{-}$potential energy curves.

\section{ACKNOWLEDGMENTS}

The authors would like to extend their sincere appreciation to the Deanship of Scientific Research at King Saud University for funding the research through the Research Group Project No. RGP-333. This work was supported by the Programme National Physique et Chimie du Milieu Interstellaire (PCMI) of CNRS/INSU with INC/INP co-funded by CEA and CNES. F.L. acknowledges the French National Research Agency (ANR) through a grant to the Anion Cos Chem Project (No. ANR-14-CE33-0013).

${ }^{1}$ D. Zhang and S. Willitsch, preprint arXiv:1703.07133 (2017).

${ }^{2}$ S. Chefdeville, Y. Kalugina, S. Y. van de Meerakker, C. Naulin, F. Lique, and M. Costes, Science 341, 1094 (2013).

${ }^{3}$ A. Bergeat, J. Onvlee, C. Naulin, A. van der Avoird, and M. Costes, Nat. Chem. 7, 349 (2015).

${ }^{4}$ J. Jankunas, K. Jachymski, M. Hapka, and A. Osterwalder, "Communication: Importance of rotationally inelastic processes in low-energy penning ionization of $\mathrm{CHF}_{3}$," J. Chem. Phys. 144, 221102 (2016).

${ }^{5}$ J. Mikosch, M. Weidemüller, and R. Wester, Int. Rev. Phys. Chem. 29, 589 (2010)

${ }^{6}$ D. Hauser, S. Lee, F. Carelli, S. Spieler, O. Lakhmanskaya, E. S. Endres, S. S. Kumar, F. Gianturco, and R. Wester, Nat. Phys. 11, 467 (2015). 
${ }^{7}$ E. Roueff and F. Lique, Chem. Rev. 113, 8906 (2013).

${ }^{8}$ M. Agúndez, J. Cernicharo, M. Guélin, C. Kahane, E. Roueff, J. Kłos, F. Aoiz, F. Lique, N. Marcelino, J. Goicoechea et al., Astron. Astrophys. 517, L2 (2010)

${ }^{9}$ P. Thaddeus, C. Gottlieb, H. Gupta, S. Brünken, M. McCarthy, M. Agúndez, M. Guélin, and J. Cernicharo, Astrophys. J. 677, 1132 (2008).

${ }^{10}$ J. Cernicharo, M. Guélin, M. Agúndez, M. McCarthy, and P. Thaddeus, Astrophys. J., Lett. 688, L83 (2008).

${ }^{11}$ J. Cernicharo, M. Guélin, M. Agúndez, K. Kawaguchi, M. McCarthy, and P. Thaddeus, Astron. Astrophys. 467, L37 (2007).

${ }^{12}$ H. Gupta, C. Gottlieb, M. McCarthy, and P. Thaddeus, Astrophys. J. 691, 1494 (2009).

${ }^{13}$ S. Brünken, H. Gupta, C. Gottlieb, M. McCarthy, and P. Thaddeus, Astrophys. J., Lett. 664, L43 (2007).

${ }^{14}$ M. Senent and M. Hochlaf, Astrophys. J. 768, 59 (2013).

${ }^{15}$ W. Chaibi, C. Delsart, C. Drag, and C. Blondel, J. Mol. Spectrosc. 239, 11 (2006).

${ }^{16}$ D. Neufeld, E. Falgarone, M. Gerin, B. Godard, E. Herbst, G. P. des Forêts, A. Vasyunin, R. Güsten, H. Wiesemeyer, and O. Ricken, Astron. Astrophys. 542, L6 (2012).

${ }^{17}$ K. M. Menten, F. Wyrowski, A. Belloche, R. Güsten, L. Dedes, and H. S. P. Müller, Astron. Astrophys. 525, A77 (2011); e-print arXiv:1009.2825.

${ }^{18}$ A. A. Penzias, P. M. Solomon, R. W. Wilson, and K. B. Jefferts, Astrophys. J., Lett. 168, L53 (1971).

${ }^{19}$ C. A. Gottlieb and J. A. Ball, Astrophys. J., Lett. 184, L59 (1973).

${ }^{20}$ C. A. Gottlieb, J. A. Ball, E. W. Gottlieb, C. J. Lada, and H. Penfield, Astrophys. J., Lett. 200, L147 (1975).

${ }^{21}$ M. Morris, W. Gilmore, P. Palmer, B. E. Turner, and B. Zuckerman, Astrophys. J., Lett. 199, L47 (1975).

${ }^{22}$ B. E. Turner, Astrophys. J., Lett. 396, L107 (1992).

${ }^{23}$ L. Gonzalez-Sanchez, F. Marinetti, E. Bodo, and F. Gianturco, J. Phys. B: At., Mol. Opt. Phys. 39, S1203 (2006).

${ }^{24}$ H.-J. Werner and P. J. Knowles, J. Chem. Phys. 82, 5053 (1985).

${ }^{25}$ P. J. Knowles and H.-J. Werner, Chem. Phys. Lett. 115, 259 (1985).

${ }^{26}$ G. Knizia, T. B. Adler, and H.-J. Werner, J. Chem. Phys. 130, 054104 (2009).

${ }^{27}$ H. Werner, P. Knowles, G. Knizia, F. Manby, M. Schtz, P. Celani, T. Korona, R. Lindh, A. Mitrushenkov, G. Rauhut et al., MOLPRo, version 2012.1, a package of ab initio programs, 2012, see http://www.molpro.net.
${ }^{28}$ R. A. Kendall, T. H. Dunning, Jr., and R. J. Harrison, J. Chem. Phys. 96, 6796 (1992).

${ }^{29}$ T. H. Dunning, Jr., J. Chem. Phys. 90, 1007 (1989).

${ }^{30}$ K. E. Yousaf and K. A. Peterson, J. Chem. Phys. 129, 184108 (2008).

${ }^{31}$ F. Lique, J. Kłos, and M. Hochlaf, Phys. Chem. Chem. Phys. 12, 15672 (2010).

${ }^{32}$ P. Halvick, T. Stoecklin, F. Lique, and M. Hochlaf, J. Chem. Phys. 135, 044312 (2011)

${ }^{33}$ Y. N. Kalugina, I. A. Buryak, Y. Ajili, A. A. Vigasin, N. E. Jaidane, and M. Hochlaf, J. Chem. Phys. 140, 234310 (2014).

${ }^{34}$ Y. Ajili, K. Hammami, N. E. Jaidane, M. Lanza, Y. N. Kalugina, F. Lique, and M. Hochlaf, Phys. Chem. Chem. Phys. 15, 10062 (2013).

${ }^{35}$ Y. Kalugina, F. Lique, and S. Marinakis, Phys. Chem. Chem. Phys. 16, 13500 (2014).

${ }^{36}$ S. F. Boys and F. Bernardi, Mol. Phys. 19, 553 (1970).

${ }^{37}$ H.-J. Werner, B. Follmeg, and M. H. Alexander, J. Chem. Phys. 89, 3139 (1988).

${ }^{38}$ G. G. Balint-Kurti, R. N. Dixon, and C. C. Marston, Int. Rev. Phys. Chem. 11, 317 (1992).

${ }^{39}$ C. C. Marston and G. G. Balint-Kurti, J. Chem. Phys. 91, 3571 (1989).

${ }^{40}$ R. LeRoy, University of Waterloo Chemical Physics Research Report CP657R, 2004.

${ }^{41}$ M. Elhanine, R. Farrenq, G. Guelachvili, and M. Morillon-Chapey, J. Mol. Spectrosc. 129, 240 (1988).

${ }^{42}$ Y. Ajili, T. Trabelsi, O. Denis-Alpizar, T. Stoecklin, A. Császár, M. M. Al-Mogren, J. Francisco, and M. Hochlaf, Phys. Rev. A 93, 052514 (2016).

${ }^{43}$ D. Papp, J. Sarka, T. Szidarovszky, A. G. Császár, E. Mátyus, M. Hochlaf, and T. Stoecklin, Phys. Chem. Chem. Phys. 19, 8152 (2017).

${ }^{44}$ J. Hutson and S. Green, Collaborative computational project, 1994.

${ }^{45}$ M. Gruebele, M. Polak, and R. J. Saykally, J. Chem. Phys. 86, 1698 (1987).

${ }^{46}$ A. Arthurs and A. Dalgarno, Proc. R. Soc. A 256, 540-551 (1960).

${ }^{47}$ D. Manolopoulos, J. Chem. Phys. 85, 6425 (1986).

${ }^{48}$ A. Faure, P. Jankowski, T. Stoecklin, and K. Szalewicz, Sci. Rep. 6, 28449 (2016).

${ }^{49}$ K. M. Christoffel and J. M. Bowman, J. Chem. Phys. 78, 3952 (1983).

${ }^{50}$ L. N. Smith, D. J. Malik, and D. Secrest, J. Chem. Phys. 71, 4502 (1979). 